\title{
Two Dimensional IIR and FIR Digital Notch Filter
}

\section{Design}

\author{
Soo-Chang Pei * and Chien-Cheng Tseng \\ Department of Electrical Engineering \\ National Taiwan University \\ Taipei, Taiwan, R.O.C.
}

\begin{abstract}
In this paper, the two dimensional (2D) digital notch filter design problem is investigated. First, we develop a simple algebraic method for $2 D$ IIR notch filter design. This approach not only has closed-form transfer function but also satisfies bounded input bunded output (BIBO) stability condition. Next, we use Lagrange multiplier method to design 2D FIR notch filters. This design is optimal in least square sense. Finally, we apply the 2D notch filter to eliminate sinusoidal or narrowband interferences superimposed on an image. Simulation results are presented to demonstrate the effectiveness of these approaches.
\end{abstract}

\section{Introduction}

In many applications of signal processing it is desired to remove narrowband or sinusoidal interferences while leaving the broadband signal unchanged. Examples are in the areas of communication, control, biomedical engineering and others. In 1D case, a typical example is to cancel $60 \mathrm{~Hz}$ power line interference in the recording of electrocardiograms(ECGS) [1]. Usually this task can be achieved by the notch filters characterized by a unit gain at all frequencies except at the sinusoidal frequencies in which their gain is zero. In 2D case, one of the image corruption examples is the occurrence of a 2D sinusoidal interference pattern (often called coherent noise) superimposed on an image [2]. The Fourier spectrum of this corrupted image exhibits a pair of symmetric peak impulses due to the sinusoidal interference, so we usually remove these impulses manually in spectrum domain and then take the inverse Fourier transform of this result to restore image [2]. This method is only suitable for off-line processing. For real-time processing purpose, a $2 \mathrm{D}$ notch filter is preferred. However, 2D notch filter design techniques have not been proposed until now. Thus it is still an open question in the filter design area. In this paper, we will present some simple approaches to design 2D IIR and FIR notch filters.

If $2 \mathrm{D}$ notch filter is considered to design as an IIR type filter, some well-known optimization techniques which have been developed to design general 2D filters can be used here [3]. However, these methods are not only time-consuming but also difficult to control stability. Because $2 \mathrm{D}$ notch filter is a special one, we may use a simple algebraic method to design it. The main feature of this approach is easy to monitor stability and have closed-form transfer function. A similar technique have been successfully used to design the special 3D IIR beam filter for enhancing a spatial plane-wave by Bruton[4]. On the other hand, if $2 \mathrm{D}$ notch filter is designed as a FIR type filter, it is basically a mininization problem with linear constraints. Therefore, many well-established optimization methods can be used. In this paper, we will use Lagrange multiplier methods to design it in the least square sense.

\section{2D IIR Notch Filter Design}

In this section, we will use simple algebraic method to design $2 \mathrm{D}$ IIR notch filter. The frequency response for a $2 \mathrm{D}$ ideal notch filter is given by

$$
H_{d}\left(e^{j \omega_{1}}, e^{j \omega_{2}}\right)= \begin{cases}0 & \left(\omega_{1}, \omega_{2}\right)=\left(\omega_{1 N}, \omega_{2 N}\right) \\ 1 & \text { otherwise }\end{cases}
$$

where $\left(\omega_{1 N}, \omega_{2 N}\right)$ is the notch frequency. Now the problem is to find a 2D IIR filter which satisfies this specification. The proposed method, as shown in Fig.1, can be divided into two simple filter designs. One is 2D parallel line filter $H_{p}\left(z_{1}, z_{2}\right)$, the other is $2 \mathrm{D}$ straight line filter $H_{s}\left(z_{1}, z_{2}\right)$. Then the desired notch filter transfer function is given by

$$
H_{N}\left(z_{1}, z_{2}\right)=1-H_{p}\left(z_{1}, z_{2}\right) H_{s}\left(z_{1}, z_{2}\right)
$$

Now, let us design filters $H_{p}\left(z_{1}, z_{2}\right)$ and $H_{s}\left(z_{1}, z_{2}\right)$ as follows: (A) 2D Parallel Line Filter:

The frequency specification of $2 \mathrm{D}$ parallel line filter is given by

$$
H_{p}\left(e^{j \omega_{1}}, e^{j \omega_{2}}\right)= \begin{cases}1 & \omega_{2}= \pm \omega_{2 N} \\ 0 & \text { otherwise }\end{cases}
$$

This special filter can be easily designed by choosing $H_{p}\left(z_{1}, z_{2}\right)$ as

$$
H_{p}\left(z_{1}, z_{2}\right)=\left.H_{B P}(z)\right|_{z=z_{2}}
$$

where $H_{B P}(z)$ is a $1 \mathrm{D}$ bandpass filter whose transfer function is given by

$$
H_{B P}(z)=\frac{1}{2}\left(1-\frac{a_{2}-a_{1} z^{-1}+z^{-2}}{1-a_{1} z^{-1}+a_{2} z^{-2}}\right)
$$

After some manipulation, we can get two important relations of $H_{B P}(z)$ as follows.

$$
\begin{aligned}
& a_{1}=\frac{2 \cos \left(\omega_{0}\right)}{1+\tan \left(\frac{B W}{2}\right)} \\
& a_{2}=\frac{1-\tan \left(\frac{B W}{2}\right)}{1+\tan \left(\frac{B W}{2}\right)}
\end{aligned}
$$

where $\omega_{0}$ is the center frequency of $H_{B P}(z)$ and $B W$ is the $3 d B$ bandwidth of $H_{B P}(Z)$. Hence, we only require to choose $\omega_{0}=\omega_{2 N}$ and let $B W$ be as small as possible, then $H_{p}\left(z_{1}, z_{2}\right)$ will be a desired design.

(B) 2D Straight Line Filter:

Designing 2D IIR digital filter by frequency transformation of 2D analog protype filter is a simple and useful method, so we will use this technique to obtain the transfer function of straight line filter $H_{s}\left(z_{1}, z_{2}\right)$. Consider the simple first-order 2D Laplace transform transfer function 


$$
T\left(s_{1}, s_{2}\right)=\frac{R}{R+L_{1} s_{1}+L_{2} s_{2}}
$$

which corresponds to the voltage transfer function of the 2D inductance-resistance network shown in Fig.2. The frequency response is given by

$$
T\left(j \Omega_{1}, j \Omega_{2}\right)=\frac{R}{R+j\left(L_{1} \Omega_{1}+L_{2} \Omega_{2}\right)}
$$

Therefore, the magnitude response $G\left(\Omega_{1}, \Omega_{2}\right)$ can be written as

$$
G\left(\Omega_{1}, \Omega_{2}\right)=\frac{R}{\left[R^{2}+\left(L_{1} \Omega_{1}+L_{2} \Omega_{2}\right)^{2}\right]^{\frac{1}{2}}}
$$

A maximum value of unity occurs in $G\left(\Omega_{1}, \Omega_{2}\right)$ at a straight line where

$$
L_{1} \Omega_{1}+L_{2} \Omega_{2}=0 \quad \text { (resonant line) }
$$

and the two $-3 \mathrm{~dB}$ lines which $G\left(\Omega_{1}, \Omega_{2}\right)=\frac{1}{\sqrt{2}}$ are

$$
L_{1} \Omega_{1}+L_{2} \Omega_{2}= \pm R \quad(-3 \mathrm{~dB} \text { lines })
$$

If we apply the double bilinear transformation

$$
s_{i}=\frac{z_{i}-1}{z_{i}+1} \quad i=1,2
$$

to $T\left(s_{1}, s_{2}\right)$, then we get the desired straight line filter as

$$
H_{s}\left(z_{1}, z_{2}\right)=\frac{1+z_{1}^{-1}+z_{2}^{-1}+z_{1}^{-1} z_{2}^{-1}}{\frac{R+L_{1}+L_{2}}{R}+\frac{R-L_{1}+L_{2}}{R} z_{1}^{-1}+\frac{R+L_{1}-L_{2}}{R} z_{2}^{-1}+\frac{R-L_{1}-L_{2}}{R} z_{1}^{-1} z_{2}^{-1}}
$$

It is obvious that a maximum value of unity occurs in $\left|H_{s}\left(e^{j \omega_{1}}, e^{j \omega_{2}}\right)\right|$ where

$$
L_{1} \tan \left(\frac{\omega_{1}}{2}\right)+L_{2} \tan \left(\frac{\omega_{2}}{2}\right)=0 \quad \text { (resonant line) }
$$

and that $\left|H_{s}\left(e^{j \omega_{1}}, e^{j \omega_{2}}\right)\right|=\frac{1}{\sqrt{2}}$ where

$$
L_{1} \tan \left(\frac{\omega_{1}}{2}\right)+L_{2} \tan \left(\frac{\omega_{2}}{2}\right)= \pm R \quad(-3 \mathrm{~dB} \text { lines })
$$

Hence, if we properly choose the parameters as

$$
\begin{aligned}
& L_{1}=\frac{1}{\tan \left(\frac{\omega_{1} N}{2}\right)} \\
& L_{2}=\frac{-1}{\tan \left(\frac{w_{2 N}}{2}\right)}
\end{aligned}
$$

and $R$ is as small as possible, then $H_{s}\left(z_{1}, z_{2}\right)$ will be a straight line filter whose resonant line pass the point $\left(\omega_{1 N}, \omega_{2 N}\right)$ exactly even though it exists bending effect due to the bilinear transformation [5]. Moreover, in order to ensure boundedinput/bounded-output (BIBO) stability of this filter, we must constrain $L_{1}$ and $L_{2}$ to be nonnegative [6]. Due to this constraint and Eq.(17), we see that notch frequency $\left(\omega_{1 N}, \omega_{2 N}\right)$ will only locate at second quadrant. However, this restriction poses no practial problem because the $2 \mathrm{D}$ input signal can be reoriented to place the signal in the correct second quadrant before filtering.

Based on the preceding discussion, we consider an example with notch frequency $\left(\omega_{1 N}, \omega_{2 N}\right)=(0.5 \pi,-0.5 \pi)$. The parameters $B W=0.001 \pi$ and $R=0.001$. Fig. 3 shows the frequency reponse plot of notch filter. It is clear that the specification is well satisfied as expected. When the parameters $R$ and $B W$ approach zero, $H_{N}\left(z_{1}, z_{2}\right)$ becomes an ideal notch filter.

\section{2D FIR Notch Filter Design}

In this section, we will use Lagrange multiplier method to design 2D FIR notch filter. In general, FIR filters have many advantages over IIR filters. For example, stability is never an issue and linear phase is simple to achieve. However, FIR filter requires larger arithmetic operations than IIR filter during implimentation.

A 2D linear phase FIR digital filter with transfer function $H\left(z_{1}, z_{2}\right)$ has a frequency response

$H\left(e^{j \omega_{1}}, e^{j \omega_{2}}\right)=e^{-j N_{1} \omega_{1}} e^{-j N_{2} \omega_{2}} \sum_{m=-N_{1}}^{N_{1}} \sum_{n=-N_{2}}^{N_{2}} h(m, n) e^{-j\left(m \omega_{1}+n \omega_{2}\right)}$

where the impulse response $h(m, n)$ satisfy the half-plane symmetric condition, i.e.

$$
h(m, n)=h(-m,-n)
$$

,and the filter length is $\left(2 N_{1}+1\right) \times\left(2 N_{2}+1\right)$. After some maniputation, it is easily shown that the magnitude response $G\left(\omega_{1}, \omega_{2}\right)$ of $H\left(z_{1}, z_{2}\right)$ is given by

$$
G\left(\omega_{1}, \omega_{2}\right)=\sum_{i=1}^{R} a(i) g_{i}\left(\omega_{1}, \omega_{2}\right)
$$

where the dimension $R=\left(N_{1}+1\right)+N_{2}\left(2 N_{1}+1\right)$ and

$$
\begin{aligned}
g_{i}(m, n) & =\cos \left(m \omega_{1}+n \omega_{2}\right) \\
a(i) & = \begin{cases}h(0,0) & i=1 \\
2 h(m, n) & i \neq 1\end{cases}
\end{aligned}
$$

For tha sake of convenience, we rewrite $G\left(\omega_{1}, \omega_{2}\right)$ as the following vector form

$$
G\left(\omega_{1}, \omega_{2}\right)=A^{t} C\left(\omega_{1}, \omega_{2}\right)
$$

in which the vectors are

$$
\begin{aligned}
A & =\left[\begin{array}{ll}
a(1) & a(2) \cdots a(R)
\end{array}\right]^{t} \\
C\left(\omega_{1}, \omega_{2}\right) & =\left[\begin{array}{ll}
g_{1}\left(\omega_{1}, \omega_{2}\right) & g_{2}\left(\omega_{1}, \omega_{2}\right) \cdots g_{R}\left(\omega_{1}, \omega_{2}\right)
\end{array}\right]^{t}
\end{aligned}
$$

Generally speaking, two most common critera used in linear phase FIR designs are the least square error and the minimax error criteria. Now, let's design $G\left(\omega_{1}, \omega_{2}\right)$ to approximate the ideal notch filter frequency response in least square error sense.

In this sense, the filter design problem can be formulated as as follows:

$$
\begin{aligned}
& \text { Minimize } \int_{-\pi}^{\pi} \int_{-\pi}^{\pi}\left|G\left(\omega_{1}, \omega_{2}\right)-1\right|^{2} d \omega_{1} d \omega_{2} \\
& \quad=\int_{-\pi}^{\pi} \int_{-\pi}^{\pi}\left(A^{t} C\left(\omega_{1}, \omega_{2}\right)-1\right)^{2} d \omega_{1} d \omega_{2} \\
& =A^{t} Q A-2 P^{t} A+1
\end{aligned}
$$

Subject to $\quad C^{t}\left(\omega_{1 N}, \omega_{2 N}\right) A=0$

where the positive definite symmetric matrix $Q$ and the vector $P$ are given by

$$
\begin{gathered}
Q=\int_{-\pi}^{\tau} \int_{-\pi}^{\pi} C\left(\omega_{1}, \omega_{2}\right) C^{t}\left(\omega_{1}, \omega_{2}\right) d \omega_{1} d \omega_{2} \\
P=\int_{-\pi}^{\pi} \int_{-\pi}^{\pi} C\left(\omega_{1}, \omega_{2}\right) d \omega_{1} d \omega_{2}
\end{gathered}
$$

Therefore, the optimal solution is given by 


$$
\begin{aligned}
A_{o p t}= & {\left[I-Q^{-1} C\left(\omega_{1 N}, \omega_{2 N}\right)\left[C^{t}\left(\omega_{1 N}, \omega_{2 N}\right) Q^{-1}\right.\right.} \\
& \left.\left.C\left(\omega_{1 N}, \omega_{2 N}\right)\right]^{-1} C^{t}\left(\omega_{1 N}, \omega_{2 N}\right)\right] Q^{-1} P
\end{aligned}
$$

Based on the preceding discussion, we will design a 2D FIR notch filter with notch frequency $\left(\omega_{1 N}, \omega_{2 N}\right)=(0.5 \pi, 0.5 \pi)$. The filter length is $11 \times 11$. Fig.4 shows the 2D frequency response of FIR notch filter. It can be seen from this result that the specification is well satisfied.

\section{Application Examples}

In this section, we will use the 2D notch filter to remove single sinusoidal interference superimposed on an image. A notable example of this periodic image degration is found in image that has been derived from electro-optical scanner[2]. This interference is usually caused by coupling and amplification of low-level signals in the electronic circuitry. All the examples are performed on the VAX 3600 computer. The images used are 512 by 512 black and white images. The intensity of each pixel is quantized into 256 gray levels ( $8 \mathrm{bbp}$ ).

The image shown in Fig.5(a) is the Lena image corrupted by a sinusoidal pattern of the form

$$
30 \sin (0.1 \pi m-0.2 \pi n)
$$

The Fourier spectrum of this image, shown in Fig.5(b), exhibit a pair of symmetric peak impulses located at $(0.1 \pi,-0.2 \pi)$ and $(-0.1 \pi, 0.2 \pi)$. We usually remove this impluse manually in the frequency domain and take the inverse Fourier transform of this result to restore image. The resultant image, shown in Fig.5(c), is clearly free from interference. Although this method is simple, it is only suitable for off-line batch processing. For real-time processing purpose, the 2D notch filter is preferred. Moreover, sinusoidal frequency may not exactly locate at the resolution grid of 2D fast Fourier tansform, so a small region around the peak impulse must be removed. This will cause some information loss of the image. In 2D notch filter case, the notch frequency can be chosen exactly the same as the sinusoidal frequency, so there is nearly no information loss. Now, we design a 2D IIR notch filter with $\left(\omega_{1 N}, \omega_{2 N}\right)=(0.1 \pi,-0.2 \pi), R=0.1$, and $B W=0.05 \pi$ to remove the interference in spatial domain. Resultant image suffers very severe transient states degradation near the two boundaries in Fig.5(d). In order to suppress this transient states due to incomplete boundary data, the algorithm developed in [7] can be used. We compute the initial condition by extending the boundary values of the given image data beyond its region of support. The resultant filtered image, shown in Fig.5(e), is free from the interference. Furthermore, we may design a 2D FIR notch filter with $\left(\omega_{1 N}, \omega_{2 N}\right)=(0.1 \pi,-0.2 \pi)$ and length $11 \times 11$ to remove sinusoidal interference. The filtered image is shown in Fig.5(f). It is clear that the interference has been removed.

Finally, we compare the computation complexity of the above three methods. For a image with size $N \times N$, the FFT method

requires $N^{3} \log _{2} N$ complex multiplications to restore an image, but IIR notch filter only require $20 \mathrm{~N}^{2}$ real multiplications. As to a $M_{1} \times M_{2}$ FIR notch filter, it requires $\frac{M_{1} M_{2}}{2} N^{2}$ real multiplications. Hence, the notch filtering technique is much more efficient than conventional FFT method in computation complexity.

\section{Conclusion}

In this paper, the $2 \mathrm{D}$ IIR and FIR digital notch filter design$s$ have been studied. The IIR notch filter is designed by very simple algebraic method, and the FIR notch filters are designed by Lagrange multiplier method. Several design and application examples have been used to illustrate the effectiveness of these approaches. However, only the fixed 2D notch filters are considered here. Thus it is interesting to develop the adaptive 2D notch filter algorithm. This topic will be investigated in the furture.

\section{References}

[1] B.Widrow and S.D.Stearns, "Adaptive Signal Processing", Englewood Cliffs, NJ: Prentice Hall 1985,

[2] R.C.Gonzalez and P.Wintz, "Digital Image Processing", Addison-Wesley, 2nd edition, pp.237-247,1987.

[3] J.S.Lim, "Two-Dimensional Signal and Image Processing", Englewood Cliffs, NJ: Prentice-Hall 1990.

[4] L.T.Bruton and N.R.Bartley, "Three-dimensional image processing using the concept of network resonance", IEEE Trans. Circuits and Syst., Vol. CAS-32, pp664-672, July 1985.

[5] S.C.Pei and S.B.Jaw, "Comments on highly selective threedimensional recursive beam filters using intersection resonant planes", IEEE Trans. Circuits and Syst., Vol. CAS-33, pp.669-670, July 1986 .

[6] P.Agathoklis and L.T.Bruton, "Practical BIBO stability of $\mathrm{N}$-dimensional discrete systems", Proc. Inst. Elect. Eng., Vol.130, pt.G, No.6, pp.236-242, Dec. 1983.

[7] W.E.Alexander, "Initial condition transient suppression for 2-dimensional recursive filters", 1984 Int'l conf. on Acoust. Speech, Signal processing, pp.2011-2014 .

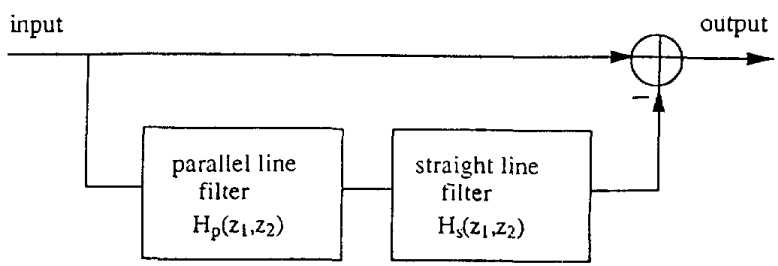

Fig.1 The block diagram for 2D IIR notch filter design. 

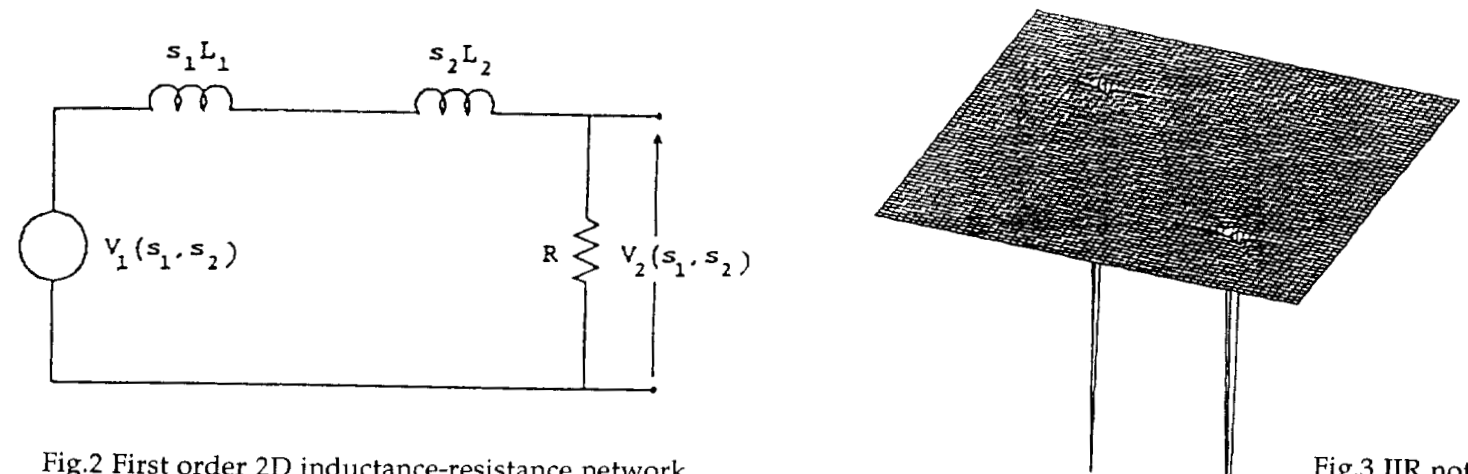

Fig.2 First order 2D inductance-resistance network.
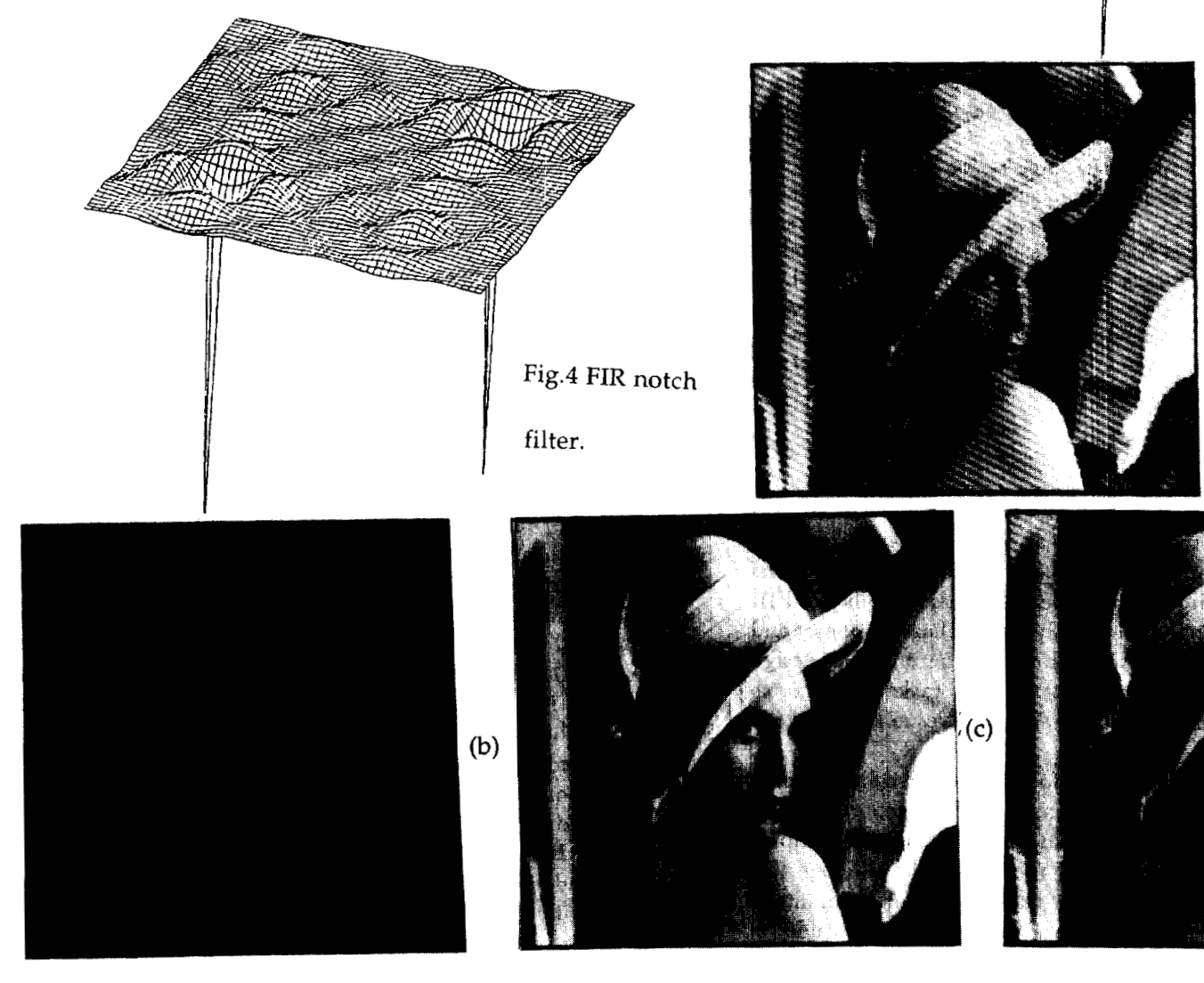

(a)

(b)
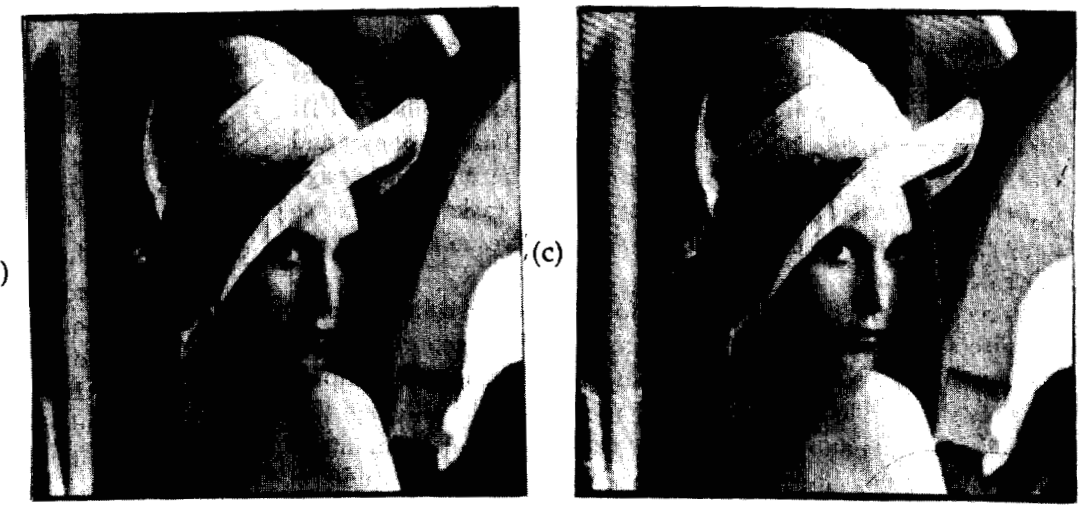

(d)
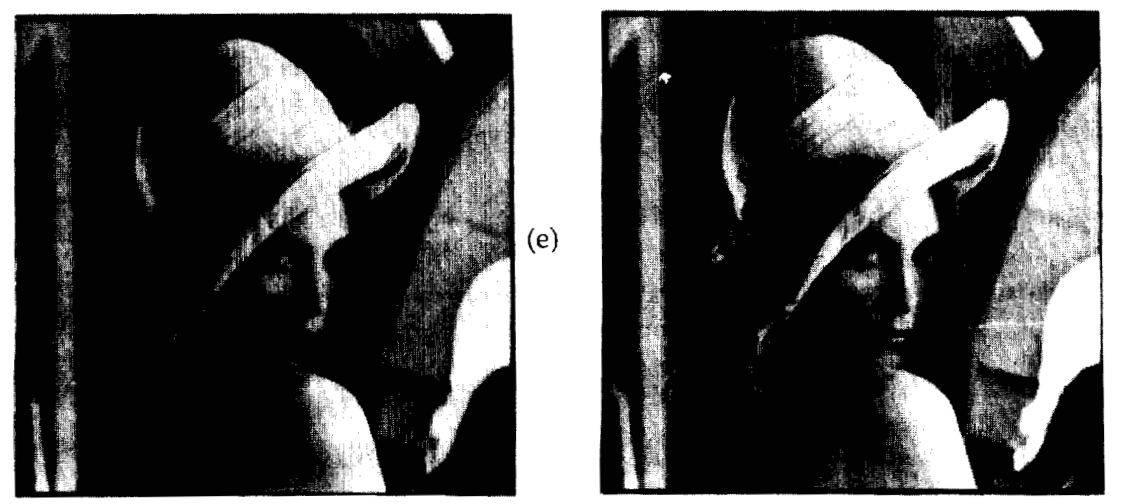

Fig, 5 Example of sinusoidal interference removal. 